\title{
Estudio comparativo entre la legislación de eficiencia energética de Colombia y España
}

Guillermo Mejía*

A Comparative Study between the Energetic Efficiency Legislation in Calombia and Spain

Étude comparative entre les légis/ations colombiennes et espagnole sur l'efficacité énergétique

Estuda comparativo da legis/ação de eficiência energética da colômbia e a espanha

"Maestría en Eficiencia Energética y Ecología Industrial.Universidad de Zaragoza, España. Máster en Administración de Negocios, Universidad Politécnica de Madrid, España Maestría en Energías Renovables, Universidad de Zaragoza, España. Especialista en Administración de Negocios, Universidad Politécnica de Madrid, España.

Especialista en Redes de Telecomunicaciones, Universidad del Valle, Cali, Colombia Especialista en Sistemas, Universidad del Valle, Cali, Colombia Ingeniero en Electrónica y Telecomunicaciones, Universidad del Cauca, Popayán, Colombia. 


\section{RESUMEN}

La mejora en la eficiencia de conversión, distribución y uso de la energía, presenta un importante potencial para mitigar los efectos del consumo energético y su impacto en el problema mundial del cambio climático. Las políticas públicas de eficiencia energética, son implementadas a través de diferentes mecanismos incluyendo regulación de precios, reglamentación o legislación e incentivos económicos y fiscales. Este trabajo se centra en la reglamentación y legislación, desde la perspectiva de dos países con niveles sociales y económicos muy diferentes: Colombia y España. Se realiza una comparación de los resultados de su aplicación, medidos con base en la intensidad energética. También, hace se hacen recomendaciones para el desarrollo de una mejor política de eficiencia en Colombia.

\section{ABSTRACT}

The efficiency in the conversion, distribution and use of energy shows a great potential to control the effects of energetic consumption and its impact on worldwide climate changes. The energetic efficiency public policies are implemented through different mechanisms such as prices, regulation and fiscal and economic incentives. This paper focuses on its regulation from the perspective of two countries with different social and economic levels, Spain and Colombia, and it also compares the results of its application, based on energetic intensity. Additionally, recommendations are made for the development of the best policies of efficiency in Colombia.

\section{RESUMÉÉ}

L'amélioration de l'efficacité d'utilisation, de conversion, et de distribution de l'énergie représente un potentiel significatif pour l'atténuation des effets sur la consommation énergétique et son impact sur le changement climatique. Des politiques d'amélioration de l'efficacité énergétique sont mises en œuvre par le biais de différents mécanismes tels que les prix, la règlementation ou les bénéfices fiscaux. Cette analyse porte sur la différence de réglementation mise en place dans deux pays possédant des niveaux de développement économiques et sociaux très différents : l'Espagne et la Colombie. Nous comparerons les résultats de ces politiques mesurés en termes d'intensité énergétique. Nous proposons également des recommandations pour la mise en place d'une plus grande efficacité énergétique en Colombie.

\section{RESUMO}

A melhoria na eficiência de conversão, distribuição e uso da energia apresenta um importante potencial para mitigar os efeitos do consumo energético e seu impacto no problema mundial da mudança do clima. As políticas públicas de eficiência energética são implementadas através de diferentes mecanismos incluindo preços, regulação e incentivos econômicos e fiscais. Este trabalho está centrado na regulação desde a perspectiva de dois países com níveis sociais e econômicos muito diferentes: a Espanha e a Colômbia, e compara os resultados de sua aplicação, medidos com base na intensidade energética. Recomendações também são feitas para o desenvolvimento de uma melhor política de eficiência na Colômbia.
Palabras clave

\author{
Eficiencia energética \\ Energía \\ Legislación \\ Regulación \\ Intensidad energética
}

\section{Keywords}

Efficiency energetic Energy

Legislation

Regulation

Energetic intensity.

\title{
Palavras-chave
}

\author{
Eficiência energética \\ Energia \\ Legislação \\ Regulação \\ Intensidade energética.
}




\section{INTRODUCCIÓN}

$\mathbf{E}$ I cambio climático ha sido reconocido por los Estados como uno de los problemas ambientales más relevantes de la época actual (Angulo, 2010; Rodríguez, 1999) y como tal, ha sido incorporado a la agenda política internacional y nacional de cada uno de ellos. Como resultado de esto, se han desarrollado políticas públicas que buscan, entre otros, mitigar las emisiones de gases de efecto invernadero asociadas al sector energético responsable de más del $60 \%$ de estas (IPCC, 2007).

Entre las estrategias adoptadas en el sector energético, tanto a nivel de generación (suministro) como a nivel de demanda (uso), está la eficiencia energética (Aranda et al., 2010; Poveda, 2007), entendida como la reducción de la energía utilizada para un determinado servicio (calefacción, iluminación, etc.) o nivel de actividad (WEC, 2010).

Las políticas públicas de eficiencia energética, incluyen todas las intervenciones públicas destinadas a mejorar la eficiencia energética de un país, a través de precios adecuados, marco institucional, leyes, regulaciones, impuestos, subsidios y suministro de información o servicios, cuya aplicación varía acorde con cada sector, tomando en cuenta sus diferentes características (Reddy et. al., 2009; WEC, 2010).

Dentro de todo este abanico de opciones, este trabajo se concentra en analizar las leyes y regulaciones como instrumentos para el desarrollo de la política de eficiencia energética, porque si bien los mecanismos de orientación de mercado han definido por años muchas de las políticas ambientales, incluyendo el manejo sustentable de la energía y la eficiencia energética, cada vez se reconoce más la importancia de intervención de los gobiernos para el éxito de las mismas (IEA, 2012).

El objetivo de los instrumentos regulatorios es obligar a los agentes económicos a adoptar ciertos comportamientos considerados beneficiosos, e impedir los perjudiciales, so pena de que en caso de que dichas normas no se cumpliesen o se quebrantasen, la administración utilizaría los instrumentos legales (multas, tribunales, policía) para castigar y garantizar el cumplimiento (Angulo, 2010)

En general, estas políticas regulatorias son normas legales relacionadas con valores mínimos de eficiencia o prácticas de eficiencia energética, junto con sistemas de información sistemática a los consumidores a través de auditorías energéticas o etiquetas; y su aplicación es más amplia en los sectores residenciales y de servicios, donde las fuerzas de mercado no dan señales adecuadas y se requieren de cambios efectivos en el comportamiento de millones de consumidores que carecen de información y recursos para actuar (WEC, 2010).

Desafortunadamente no todos los países han avanzado de manera similar en la legislación relacionada con la eficiencia energética. La Unión Europea y sus países miembros incluyendo España, han adoptado un marco relativamente exigente frente al tema mientras que Colombia poco o nada ha avanzado en la misma materia.

El objetivo de este trabajo es revisar la situación actual de ambos marcos legislativos, evaluar los resultados obtenidos y sugerir recomendaciones para la mejora del mismo en Colombia. 


\section{LA LEGISLACIÓN EN COLOMBIA}

L a Ley 697 de 2001 mediante la cual se fomenta el uso racional y eficiente (URE) de la energía establece el marco jurídico general para el desarrollo de todas las normas posteriores en eficiencia energética en Colombia.

Esta Ley creó el Programa de Uso Racional y Eficiente de la energía y demás formas de energía no convencionales (PROURE), el cual fue reglamentado por el Decreto 3683 de 2003 y cuyo objeto es "aplicar gradualmente programas para que toda la cadena energética este cumpliendo permanentemente con los niveles mínimos de eficiencia energética...".

Solo en 2010 se adoptó el Plan de Acción Indicativo 2010-2015 para desarrollar el PROURE, y por primera vez, se definieron objetivos específicos, subprogramas estratégicos y prioritarios, y metas del ahorro (Tabla 1). El potencial de ahorro de energía eléctrica fue estimado por la UPME y las metas fueron adoptadas con base a reducciones de consumo anuales a partir de 2010.

Tabla 1. Potenciales y metas de ahorro a 2015.

\begin{tabular}{|l|c|c|c|c|}
\hline \multicolumn{1}{|c|}{ SECTOR } & $\begin{array}{c}\text { Electricidad } \\
\text { (GWh/año) }\end{array}$ & \multicolumn{3}{c|}{$\begin{array}{c}\text { Otros Energéticos } \\
\text { (Tcal/año) }\end{array}$} \\
\hline & Potencial \% & Meta \% & Potencial \% & $\begin{array}{c}\text { Meta } \\
\%\end{array}$ \\
\hline A nivel nacional & 20,3 & 14,75 & N/D & 2,1 \\
\hline Residencial & 10,6 & 8,66 & N/D & 0,55 \\
\hline Industrial & 5,3 & 3,43 & N/D & 0,25 \\
\hline $\begin{array}{l}\text { Comercial, Público y } \\
\text { Servicios }\end{array}$ & 4,6 & 2,66 & N/D & N/D \\
\hline Transporte & N/A & N/A & $\begin{array}{c}0,44^{*} \\
1,06 * *\end{array}$ & 0,33 \\
& & & & 0,96 \\
\hline
\end{tabular}

* Potencial estimado con base en reconversión tecnológica.

** Potencial estimado con base en buenas prácticas de conducción.

Fuente. Elaboración propia del autor.

Derivado de la Ley de URE, se han desarrollado los reglamentos técnicos para instalaciones eléctricas (RETIE), de iluminación y alumbrado público (RETILAP) y se ha proyectado el reglamento de etiquetado (RETIQ). Como complemento a la legislación, se han otorgado incentivos fiscales para el desarrollo de ciertos proyectos de eficiencia energética.Sin embargo, en otras áreas se ha avanzado poco:
- Sobre cogeneración existe un marco legal de perfeccionamiento más bien reciente, pero su desarrollo ha sido casi nulo tal vez por las restricciones para la venta de la energía excedente.

- Los sectores y áreas donde no existe en la actualidad legislación son: edificios, etiquetado, requerimientos sobre desempeño energético de electrodomésticos y equipos, transporte e industria. 
- En Colombia tampoco se cuenta con una agencia nacional de eficiencia energética capaz de evaluar, coordinar y hacer cumplir las políticas ${ }^{9}$.

\subsection{La Ley 1715 de 2014}

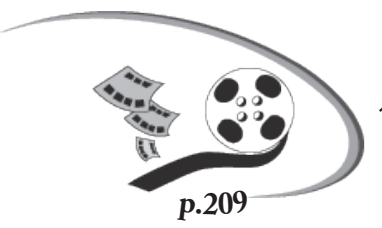

El pasado 13 de mayo de 2014, el Gobierno Nacional expidió la Ley 1715 por medio de la cual se regula la integración de las energías renovables

no convencionales al sistema energético nacional.

El Capítulo $V$ de esta Ley reafirma la necesidad de desarrollar el PROURE a través de:

- Planes de acción indicativos.

- Reglamentaciones técnicas.

- Sistemas de etiquetado e información al consumidor.
Sobre los planes indica que "responderá (sic) a una estructura sectorial que permita fijar objetivos específicos para los diferentes sectores o consumidores finales y contendrá una relación de medidas e instrumentos para su ejecución en cada uno de los sectores identificados"; y promete que "para el desarrollo del PROURE se dotará adecuadamente de los medios financieros necesarios para la consecución de los objetivos aprobados...".

Por último ordena que "para el adecuado seguimiento y evaluación del cumplimento de los objetivos" se realizará, cada cuatro años, una evaluación de "los planes y programas de ahorro para la gestión eficiente de la energía".

Esta Ley espera su reglamentación, desarrollo e implementación.

\section{LA LEGISLACIÓN EN ESPAÑA}

E n España la legislación sobre eficiencia energética deriva de las políticas energética y medioambiental de la Unión Europea a través de los planes de acción comunitarios (el último del año 2011), y de las directivas sobre uso final de la energía (Directiva 2012/27/UE vigente respecto a las Directivas

2006/32/CE y 93/76/CEE), sobre eficien-cia energética en edificios (Directiva 2010/31UE), cogeneración (Directiva 2004/8/CE derogada con efecto desde el 5 de junio de 2014 por la Directiva 2012/27/UE), diseño ecológico (Directiva 2009/125/CE) y etiquetado energético (Directiva 2010/30/UE).
Este marco político y jurídico se traslada al ordenamiento legal español y tiene su desarrollo a través de los Planes de Acción de Eficiencia Energética, (PAEE), el último de los cuales corresponde al período 2011-2020.

Un PAEE incluye un conjunto de medidas y mecanismos en aras de unos objetivos de ahorro previstos. Los mecanismos de actuación son básicamente, normativos y de apoyo. Los resultados son evaluados periódicamente con base en indicadores preestablecidos.

Estos planes se ejecutan bajo la coordinación y supervisión del Instituto para la Diversificación y Ahorro de la Energía (IDEA).

\footnotetext{
${ }^{9}$ Según el World Energy Council (2010) "una agencia de eficiencia energética se define como un cuerpo con una fuerte capacidad técnica, dedicada a la aplicación de la política nacional de eficiencia energética (en algunos casos también se encarga de la política ambiental)." En Colombia, la Unidad de Planeación Minero Energética (UPME), entidad técnica adscrita al Ministerio de Minas y Energía, tiene entre sus funciones "fomentar, diseñar y establecer los planes, programas y proyectos, relacionados con el uso eficiente, ahorro y conservación de la energía..." (Decreto 1258 de 2013).

Estas funciones son más de planeación y apoyo técnico que de aplicación y control de los programas, por lo cual no podemos considerarla una "agencia de eficiencia energética".
} 
Los sectores específicos también son objeto de legislación aplicada. Por ejemplo, en el sector de edificios residenciales y comerciales se cuenta con Código Técnico de la Edificación (CTE), el Reglamento de Instalaciones térmicas en Edificios (RITE) y la certificación energética de edificios.
También existen incentivos económicos (que en su momento impulsaron la cogeneración de manera significativa) y permisos de emisión negociables para ciertos sectores industriales y las empresas de energía.

\section{COMPARACIÓN}

Se relacionan en forma comparativa las medidas legislativas para la eficiencia energética de España y Colombia (Tabla 2).

Tabla 2. Comparación de la legislación de eficiencia energética de España y Colombia.

\begin{tabular}{|c|c|c|}
\hline POLÍTICA & ESPAÑA & COLOMBIA \\
\hline \multicolumn{3}{|l|}{ MARCO LEGISLATIVO } \\
\hline Legislación general & $\begin{array}{l}\text { Directiva de Eficiencia Energética } 2012 / 27 / \\
\text { UE vigente, respecto a las Directivas } \\
\text { 2006/32/CE y 93/76/CEE. }\end{array}$ & $\begin{array}{l}\text { Ley } 697 \text { de } 2001 \text { sobre el uso racional y } \\
\text { eficiente de la energía. }\end{array}$ \\
\hline Agencia de eficiencia & $\begin{array}{l}\text { Instituto para la Diversificación y Ahorro de } \\
\text { la Energía (IDAE). }\end{array}$ & No existe. \\
\hline Planes de acción & $\begin{array}{l}\text { Planes de Acción en Eficiencia Energética } \\
\text { (PAEE) obligatorios }\end{array}$ & $\begin{array}{l}\text { Programa de Uso Racional y Eficiente de } \\
\text { Energía y demás Formas de Energía No } \\
\text { Convencionales, PROURE }\end{array}$ \\
\hline Objetivos de ahorro & $\begin{array}{l}\text { Objetivo para reducir un } 20 \% \text { del consumo } \\
\text { de energía primaria para el } 2020 \text { (con } \\
\text { respecto a las previsiones de consumo } \\
\text { energético para el mismo año realizadas en } \\
\text { 2005). }\end{array}$ & $\begin{array}{l}\text { Objetivos globales a } 2015 \text { (14,75\% en } \\
\text { electricidad y } 2,1 \% \text { en otros energéticos), } \\
\text { con metas sectoriales y ahorros anuales a } \\
\text { partir de } 2010 \text { (Resolución } 180919 \text { de } 2010)\end{array}$ \\
\hline \multicolumn{3}{|l|}{ EDIFICIOS } \\
\hline $\begin{array}{l}\text { Requerimientos de desempeño } \\
\text { energético en edificios }\end{array}$ & $\begin{array}{l}\text { Código Técnico de la Edificación CTE (Real } \\
\text { Decreto } 31472006 \text { y 1371/2007) }\end{array}$ & No existe \\
\hline Certificado energético & Real Decreto 235/2013. & No existe \\
\hline Objetivo de ahorro & $\begin{array}{l}\text { Documento Básico de Ahorro de Energía } \\
\text { del CTE }\end{array}$ & No existe \\
\hline $\begin{array}{l}\text { Requerimientos de } \\
\text { instalaciones térmicas }\end{array}$ & $\begin{array}{l}\text { Reglamento de Instalaciones Térmicas RITE } \\
\text { (Real Decreto } 1027 / 2007 \text { y } 1826 / 2009 \text { ) }\end{array}$ & No existe \\
\hline \multicolumn{3}{|l|}{ ILUMINACIÓN } \\
\hline $\begin{array}{l}\text { Requerimientos de desempeño } \\
\text { energético }\end{array}$ & $\begin{array}{l}\text { Documento Básico HE.3 para iluminación } \\
\text { interior y Reglamento de eficiencia ener- } \\
\text { gética en instalaciones de alumbrado } \\
\text { exterior (Real Decreto } 1890 / 2008 \text { ) }\end{array}$ & $\begin{array}{l}\text { Reglamento Técnico para Iluminación y } \\
\text { Alumbrado Público RETILAP (Resolución } \\
181331 \text { de } 2009 \text { y modificaciones). }\end{array}$ \\
\hline
\end{tabular}


Tabla 2. Comparación de la legislación de eficiencia energética de España y Colombia (Continuación)

\begin{tabular}{|c|c|c|}
\hline POLITICA & ESPAÑA & COLOMBIA \\
\hline Retiro de lámparas & $\begin{array}{l}\text { Retiro de lámparas incandescentes y algu- } \\
\text { nas halógenas (Reglamento 244/2009/CE). }\end{array}$ & \begin{tabular}{|l|l} 
Retiro de lámparas incandescentes hasta 2013 \\
(Decreto 2331 de 2007 y modificaciones). \\
\end{tabular} \\
\hline $\begin{array}{l}\text { Reglamentación sobre diseño } \\
\text { ecológico. }\end{array}$ & Reglamento 245/009/CE & \\
\hline Reglamento 1194/2012/UE & No existe & \\
\hline $\begin{array}{l}\text { Reglamentación sobre } \\
\text { etiquetado. }\end{array}$ & Reglamento 874/2012/UE & No existe \\
\hline \multicolumn{3}{|l|}{ ELECTRODOMÉSTICOS Y EQUIPOS } \\
\hline $\begin{array}{l}\text { Requerimientos de desempeño } \\
\text { energético. }\end{array}$ & Diseño Ecológico (Real Decreto 187/2011). & No existe \\
\hline Etiquetado energético & $\begin{array}{l}\text { Etiquetas obligatorias algunos electrodo- } \\
\text { mésticos, luces y equipos (Real Decreto } \\
\text { 1390/2011). }\end{array}$ & En desarrollo. Etiquetado voluntario. \\
\hline \multicolumn{3}{|l|}{ TRANSPORTE } \\
\hline $\begin{array}{l}\text { Estándares de Ahorro de } \\
\text { Energía }\end{array}$ & $\begin{array}{l}\text { Para autos (Reglamento 443/2009/CE) y } \\
\text { vehículos comerciales livianos (Reglamento } \\
510 / 2011 / \text { U). No para camiones. }\end{array}$ & No existe \\
\hline $\begin{array}{l}\text { Etiquetado de Economía de } \\
\text { Combustible }\end{array}$ & $\begin{array}{l}\text { Autos: si; camiones: no (Real Decreto } \\
837 / 2002 \text { y Reglamento } 1222 / 2009 / \text { CE). }\end{array}$ & No existe \\
\hline Incentivos fiscales & $\begin{array}{l}\text { Renovación automóviles a través de incen- } \\
\text { tivos financieros. }\end{array}$ & No existe \\
\hline \multicolumn{3}{|l|}{ INDUSTRIA } \\
\hline $\begin{array}{l}\text { Regulación de eficiencia } \\
\text { energética. }\end{array}$ & No existe & No existe \\
\hline MEPS para motores eléctricos & $\begin{array}{l}\text { IE3 para motores trifásicos }<7,5 \mathrm{~kW} \text { en } \\
\text { 2015; todos IE3 (IE2 + variador de velocidad) } \\
\text { en } 2017 \text { (Reglamento 640/2009/CE). }\end{array}$ & Voluntaria \\
\hline \multicolumn{3}{|l|}{ SUMINISTRO ENERGÉTICO } \\
\hline Cogeneración & Directiva 2004/8/CE & Resolución 005 de 2011 \\
\hline Ahorro obligatorio & No existe & No existe \\
\hline \multicolumn{3}{|l|}{ INSTRUMENTOS ECONÓMICOS } \\
\hline Sector industrial y comercial. & $\begin{array}{l}\text { Incentivos financieros y permisos de emisión } \\
\text { negociables para el sector eléctrico y ciertas } \\
\text { industrias. }\end{array}$ & $\begin{array}{l}\text { Medidas fiscales limitados a los planes } \\
\text { indicativos del PROURE. }\end{array}$ \\
\hline Sector residencial y particulares. & $\begin{array}{l}\text { Planes de incentivos para renovación (elec- } \\
\text { trodomésticos, vehículos, etc.). }\end{array}$ & No existe \\
\hline
\end{tabular}

Fuente. Elaboración propia de los autores. 


\section{LOS RESULTADOS}

$\mathbf{L}$ a Intensidad Energética (IE), calculada como el cociente entre el consumo de energía y el Producto Interno Bruto (PIB), es la forma habitual de medir la eficiencia energética.

Se muestra la evolución de la IE final en Colombia con una tendencia descendente continua, lo cual se debe entre otros factores, a la urbanización y modernización, lo que conduce a la disminución del uso de la leña, y a su sustitución por energéticos más efi-cientes, reflejándose en una disminución del consumo expresado en energía neta; a los programas de gestión de la demanda de energía, junto a una importante penetración del gas; el desarrollo del sector eléctrico y su alta eficiencia por la gran participación de las hidroeléctricas; y al cambio en la estructura económica del país, entre otros (Prías, 2010; UPME, 2007) (Figura 1).

Figura 1. Intensidad Energética Final de Colombia [Gcal/M Col\$].

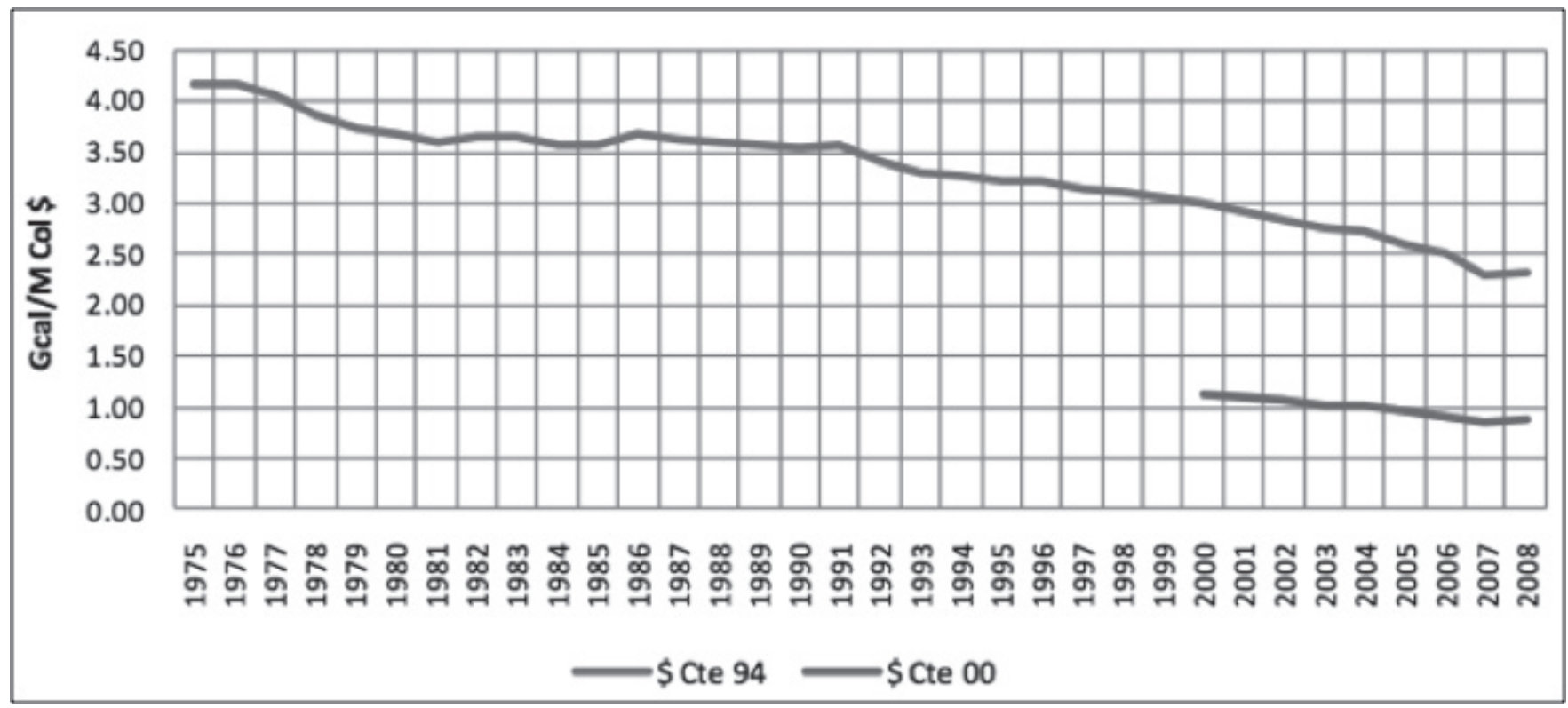

Fuente. Prías, 2010.

Sin embargo, aunque la evolución del indicador de intensidad energética muestra una variación hacia un escenario eficiente y productivo, no existen estudios ni información concluyentes, que permitan el análisis certero sobre las causas de este comportamiento, ni indicadores para el seguimiento de los objetivos de cualquier plan o política de ahorro (UPME, 2007).
Se evidencia la evolución de la IE final en España destacándose dos fases muy diferenciadas (ver Figura 2). En una primera, entre 1995 y 2004, la IE creció constantemente como producto de la expansión económica del país. A partir de este año, se mantiene una mejora constante del indicador propiciado, entre otros, por la puesta en marcha de los PAEE aunque reforzada por la crisis financiera de 2008 (Ministerio de Industria, Energía y Turismo, 2012). 
Figura 2. Intensidad Energética Final de España [tep/ MEuros de 2000]

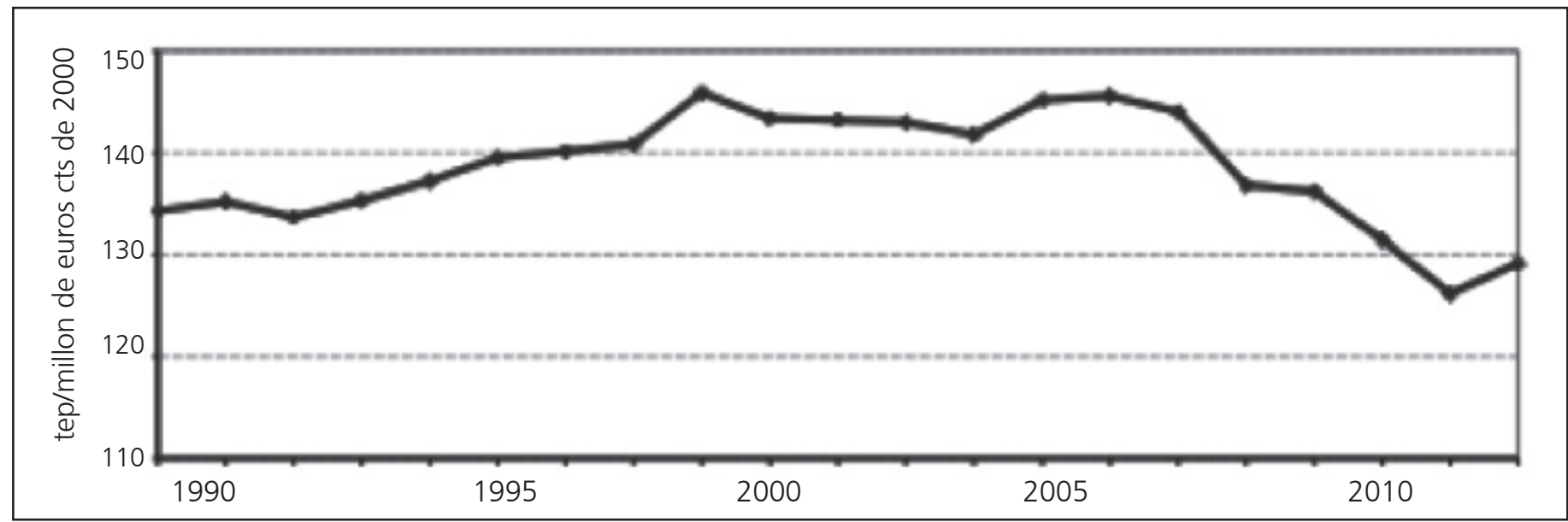

Fuente. Mendiluce y Linares (s.f.).

Se muestra la variación de la intensidad energética con paridad de poder adquisitivo (USD del año 2005) (Tabla 3). Se observa que la intensidad de energía primaria a nivel mundial se ha reducido como resultado del efecto combinado de precios de la energía, programas de eficiencia energética y, más recientemente, políticas de reducción de $\mathrm{CO}_{2}$ y otros factores económicos, tales como la tercerización de las economías (WEC, 2010).

La comparación de la IE primaria refleja una mejora mayor en Colombia frente a España (2,0\% anual frente a 0,7\% anual en el período 1990-2011; 1,9\% anual frente a 1,6\% anual en el período 2000-2011) (WEC, 2013).

Tabla 3. Variación de la intensidad energética [en ktep/ USD05p]

\begin{tabular}{|l|lr|ll|}
\hline & $\begin{array}{l}1990 / 11 \\
\text { anual }\end{array}$ & $(\%$ & $\begin{array}{l}2000 / 11 \\
\text { anual }\end{array}$ & $(\%$ \\
\hline Mundo & $-1,3$ & $-1,1$ & \\
\hline España & $-0,7$ & $-1,6$ \\
\hline Colombia & -2.0 & $-1,9$ & \\
\hline
\end{tabular}

Fuente. WEC, 2013.
Este resultado no deja de ser paradójico al comparar los esfuerzos relativos a las políticas de eficiencia energética. $Y$ aunque a priori se podría concluir que Colombia "lo ha hecho mejor" que España, la justificación de este mayor avance en el indicador puede estar en dos aspectos: por un lado, España no logró mejoras en el indicador en el período 1990-2004 como resultado de la expansión económica derivada del ingreso a la UE; y por otro Colombia, como país en desarrollo, tiene mayores oportunidades de aumentar la eficiencia energética con políticas y programas de bajo coste y fácil implementación, v. gr. la sustitución de combustibles (Buñuel, 2006).

Por tanto, dicha mejora no puede atribuirse a un mayor "éxito" de la política de eficiencia energética de Colombia (de hecho, si calculamos la variación anual de IE primaria para el periodo 2005-2011, obtenemos una disminución del 2,8\% para España y apenas un 1,4\% para Colombia). 


\section{CONCLUSIONES}

E s importante entender que nuestras conclusiones son válidas partiendo de que estamos comparando dos países con desarrollos económicos, políticos, energéticos e incluso sociales, diferentes.

España es un país desarrollado, miembro de la Unión Europea, una organización política y administrativa supranacional con una marcada conciencia social sobre los problemas ambientales y el desarrollo sostenible, que han derivado en unas políticas ambiental y energética integradas y con una alta dependencia de fuentes de energía del exterior.

Colombia por su parte, es un país en desarrollo, autosuficiente en materia energética, que dispone de una amplia legislación medioambiental promulgada que demostraría interés en el tema si no fuera por la poca efectividad de la misma, donde se considera más necesario resolver los problemas relacionados con las demandas básicas de la población en el corto plazo, es decir, un país en que todo queda subordinado al objetivo inmediato de crecimiento (Angulo, 2010).

La eficiencia energética en España responde a una política comunitaria que integra energía, medio ambiente y mitigación del cambio climático, que se traduce en el establecimiento de un "programa de eficiencia energética" con directrices, metas, tácticas y estrategias, análisis periódicos, medidas específicas por sectores y mecanismos de evaluación, todo bajo la coordinación y responsabilidad de un organismo con capacidad técnica y económica para ejecutar dicho programa. Lo anterior complementado con una legislación que crea las condiciones de mercado apropiadas para la introducción de equipos, vehículos y edificios más eficientes, y que guía la elección del consumidor hacia dichas soluciones.

No obstante en los resultados obtenidos (especialmente a partir de 2004 en que se inició el primer plan), se observa que persisten problemas estructurales en el sector energético y medio ambiental español: las emisiones en 2008 superan en un 40\% a las de 1990, frente al objetivo establecido del $15 \%$ y el $80 \%$ de la energía primaria sigue siendo importada (Mendiluce y Linares, s.f.).

Colombia basa su política en la Ley 697 de 2001 que convirtió la Gestión Eficiente de la Demanda y Uso Racional de la Energía (URE), en estrategias básicas para garantizar la satisfacción de las necesidades energéticas. $Y$ aunque la tendencia del indicador de intensidad energética en el país ha sido a la baja en una proporción aún mayor que en España, la causa de esta tendencia no se encuentra en un exitoso desarrollo de esta ley, sino en causas ajenas a la política de eficiencia energética como son la sustitución de combustibles, la modernización del país y cambios estructurales en los sectores económicos.

De hecho, se puede afirmar que los resultados de los programas de eficiencia energética han sido pocos, especialmente por la escasa atención del gobierno al desarrollo de políticas exitosas, tal vez como consecuencia del modelo energético implementado y del perfil netamente exportador de energía del país (UPME, 2007).

Entre las situaciones que afectan el adecuado desarrollo de las políticas de eficiencia energética en Colombia se pueden considerar:

- No existe un marco legal adecuado para el desarrollo y promoción de la Ley 697 sobre eficiencia energética (Consorcio Bariloche - BRP, 2007).

- No se han implementado los mecanismos necesarios para asegurar el cumplimiento y hacer seguimiento a las políticas planteadas y a la legislación expedida.

- La política de eficiencia energética no ha formado parte de un programa de políticas públicas sino que ha respondido a acciones aisladas. De hecho, hasta hace poco (2010), el programa PROURE (creado en 
2001) no contaba con un plan específico. Y este aún es limitado en objetivos y medidas.

- La falta de una entidad nacional fuerte, autónoma, respetable técnicamente y con recursos, para ejecutar programas de eficiencia energética (Poveda, 2007).

- El Gobierno ha considerado que el desarrollo de la eficiencia energética (y de las energías renovables) se logra solo con la señal económica de precios, sin considerar costos ambientales ni otros fallos del mercado.
- Se han desaprovechado oportunidades como la implementación de esquemas de etiquetado obligatorio.

Por tanto cabe resaltar que la evolución positiva de la intensidad energética experimentada en Colombia en los últimos años, no debe invitar a la complacencia, ya que responde más a

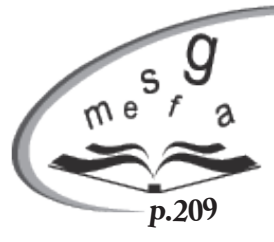
situaciones coyunturales que al resultado de exitosas políticas públicas.

\section{RECOMENDACIONES}

Del análisis realizado se desprenden dos puntos importantes:

- El éxito (relativo) de la política española para eficiencia energética se basa en la implantación de un "programa de eficiencia energética".

- Y más importante aún, este "programa de eficiencia energética" se enmarca dentro de compromisos políticos más ambiciosos de cumplimiento de acuerdos internacionales sobre cambio climático y en general, dentro de un modelo de desarrollo más sostenible.

De lo anterior se siguen tres consideraciones importantes para que Colombia pueda desarrollar, de manera efectiva y eficaz, una política de eficiencia energética:

- Es ineludible definir un modelo de desarrollo sostenible para el país que integre los aspectos económicos, medioambientales, energéticos y sociales.
- Es necesario entender que el desarrollo de una política de eficiencia energética requiere de un conjunto de mecanismos de implementación, incluyendo un marco legal adecuado, que prevalezca sobre la fuerza del mercado y la encauce.

- La implementación de esta política debe basarse en tres pilares:

- Un plan con metas y mecanismos específicos para todos y cada uno de los sectores económicos.

- Una entidad con capacidad legal, técnica y económica para liderar ese plan.

- Un sistema de información que permita identificar posibilidades de ahorro, plantear esquemas eficaces y evaluar los resultados obtenidos. 


\section{REFERENCIAS BIBLIOGRÁFICAS}

Angulo, A. (2010). Los permisos de emisión negociables como instrumento de política ambiental y su aplicación en España. Revista Desarrollo Local Sostenible. Grupo Eumed.net y Red Académica Iberoamericana Local Global, 3,(7), Recuperado de http://www.eumed.net/ rev/delos/07/

Aranda, A., Barrio, F., Zabala, I. \& Díaz, S. (2010). Técnicas para la elaboración de auditorías energéticas en el sector industrial. España: Prensas Universitarias de Zaragoza.

Buñuel, M. (2006). Tributos medioambientales y permisos de emisión negociables como incentivo a la eficiencia energética. Institución: Enteco Consulting, SRL, Comunicación Técnica en el 80 Congreso Nacional de Medio Ambiente (CONAMA): Cumbre del Desarrollo Sostenible.

Consorcio Bariloche (BRP). (2007). Consultoría para la formulación estratégica del plan de uso racional de energía y de fuentes no convencionales de energía 2007-2025. Bogotá: UPME.

International Energy Agency IEA. (2012) World Energy Outlook 2012. Francia: OECD/IEA, Francia.

Mendiluce, M. \& Linares, P. (s.f.). Análisis de la evolución de la intensidad energética en España. Economics for Energy, Vigo. Recuperado de http://www.eforenergy.org.

Ministerio de Industria, Energía y Turismo. (2011). La energía en España 2010. Madrid, España.

Ministerio de Industria, Energía y Turismo. (2012). La energía en España 2011. Madrid, España.

Panel Intergubernamental sobre el Cambio Climático IPCC. (2007). Cambio climático 2007: Informe de síntesis. Contribución de los Grupos de trabajo I, II y III al Cuarto Informe de evaluación del Grupo Intergubernamental de Expertos sobre el Cambio Climático. IPCC, Ginebra, Suiza.

Poveda, M. (2007). Eficiencia energética: recurso no aprovechado. Organización Latinoamericana de Energía OLADE, Quito, Ecuador.

Prias, O. (2010). Programa de Uso Racional y Eficiente de Energía y Fuentes No Convencionales - Proure. Informe Final. Plan de Acción 2010-2015. Bogotá: Ministerio de Minas y Energía.

Reddy, S., Assenza, G., Assenza, D. \& Hasselmann, F. (2009). Energy efficiency and climate change: conserving power for a sustainable future. New Dheli: Sage Publications India Pvt Ltd.

Rodríguez, H. (1999). Alternativas tecnológicas en el uso de la energía. La visión de los países en desarrollo. Saravia, C. \& Guerrero, E. (Eds.). Energías para un desarrollo sostenible. Ensayos sobre gestión ambiental de los recursos energéticos. Bogotá: Fondo FEN Colombia, Bogotá. 
Unidad de Planeación Minero-Energética (UPME). (2007). Plan Energético Nacional 2006-2025. Bogotá: Contexto y Estrategias. Bogotá, Colombia.

World Energy Council (WEC) (2010). Eficiencia Energética: una receta para el éxito. Londres.

World Energy Council (WEC). (2013). Energy Efficiency Indicators. Recuperado en http://www.worldenergy.org/ data/efficiency-indicators/ consultado en 28-08-2013.

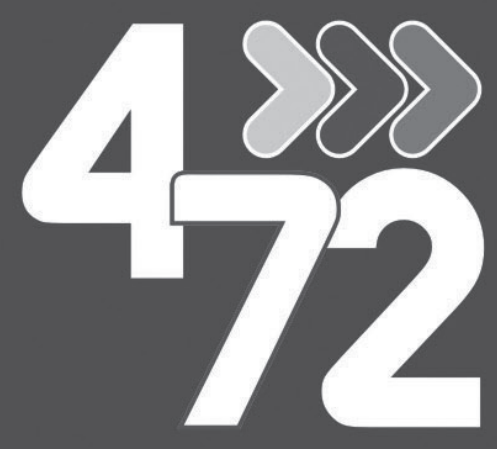

LARED POSTAL DE COLOMBIA

$$
w w w .4-72 \cdot c 0 m \cdot c 0
$$

\section{$\gamma$ Línea de Atención al Cliente Nacional 018000111210}




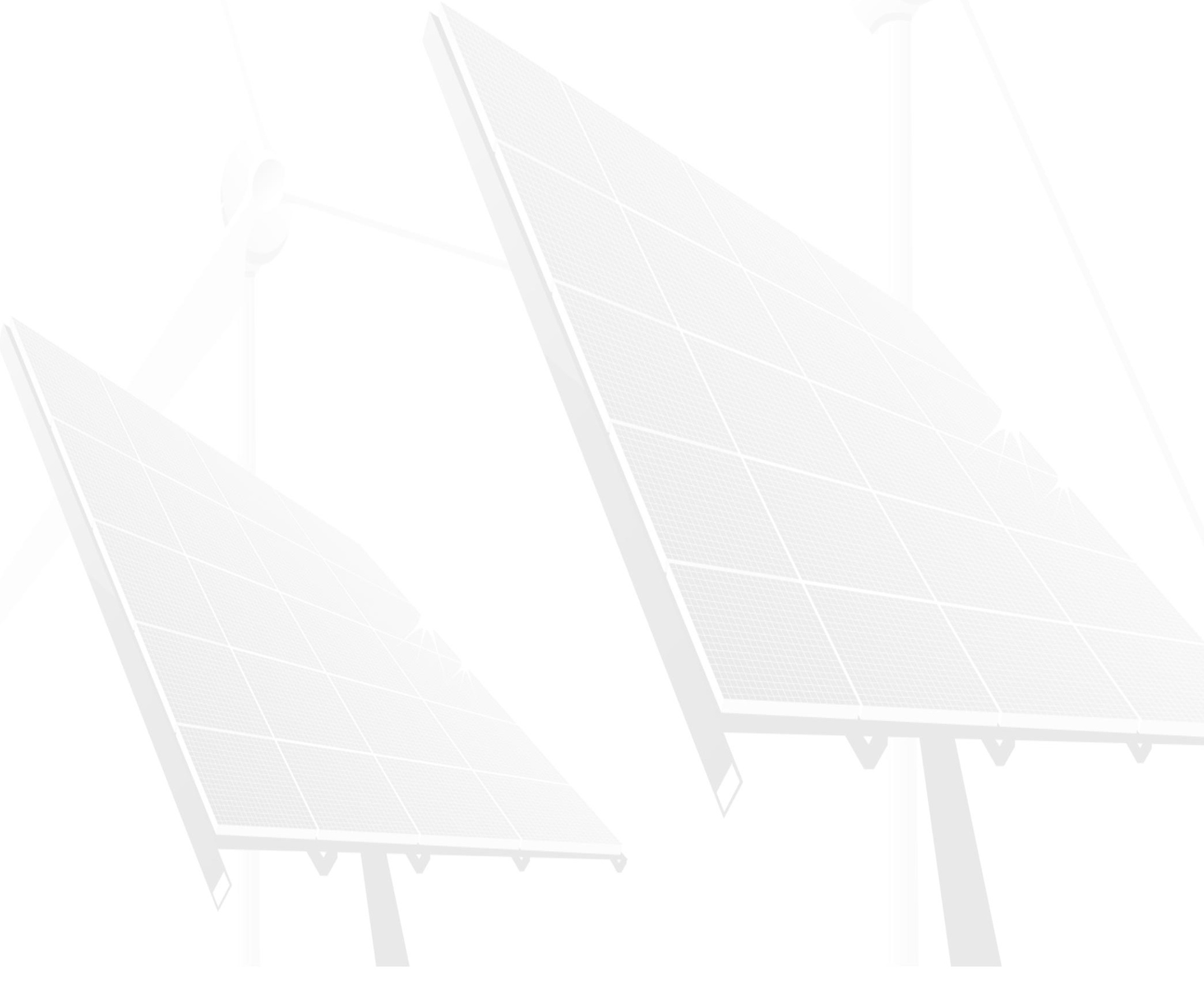

